\title{
Diagnosis and management of sinusitis
}

\author{
Kathryn L Evans
}

Sinusitis is an important source of morbidity and loss of income and may exacerbate chest disease. ${ }^{1-3}$ Most uncomplicated acute sinusitis is managed in Britain in primary care, whereas most chronic sinusitis is managed by rhinologists. Patients commonly seek advice on recurring symptoms of rhinorrhoea, congestion, intermittent facial pain, and postnasal drip, and it is important to distinguish chronic sinusitis from rhinitis.

The course and character of sinusitis are influenced by (a) repeated episodes of mucosal oedema and hypersecretion in response to infection or allergy; and (b) anatomical variants which may impair sinus ventilation and mucociliary clearance. A broader understanding of these factors helps to direct medical management either alone or with surgery. ${ }^{4}$

\section{Paranasal sinuses}

The paranasal sinuses comprise four paired cavities: the frontal, maxillary, ethmoid, and sphenoid sinuses. Each is lined with ciliated pseudostratified columnar epithelium and has a narrow ostium that opens into the nasal cavity. The ostia of the frontal, maxillary, and anterior ethmoid sinuses open into the ostiomeatal complex, which lies in the middle meatus, lateral to the middle turbinate. The posterior ethmoid and sphenoid sinuses open into the superior meatus and sphenoethmoid recess respectively. The anatomy is variable and has been extensively reviewed. ${ }^{5}$

A protective mucous blanket, which envelops bacteria and other irritants, covers the respiratory

Gloucester Royal Hospital, Gloucester GL1 3NN

Kathyrn L Evans, consultant ear, nose, and throat surgeon

$B M 7$ 1994;309:1415-22

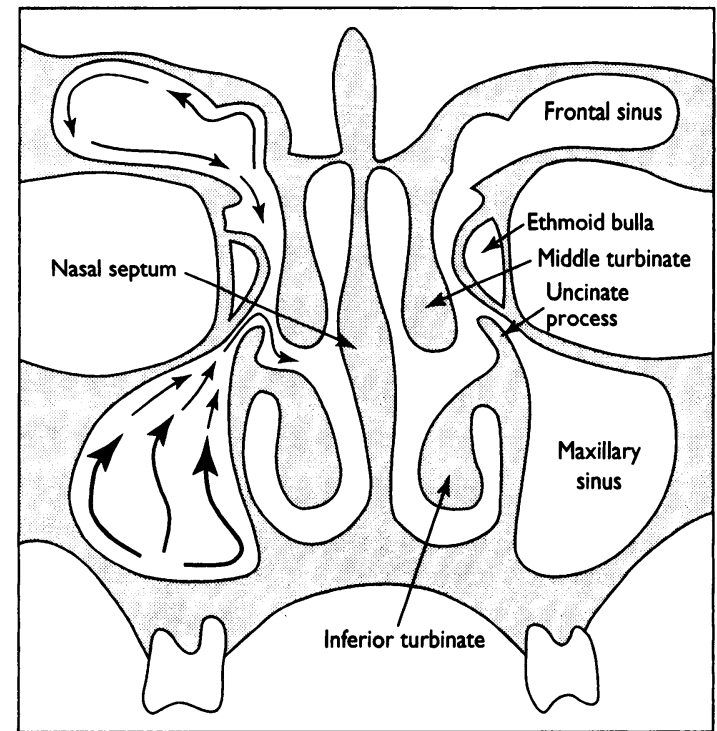

FIG 1-Mucociliary pathways in the maxillary and frontal sinuses

Summary points
- Sinusitis is an important source of morbidity
and loss of income
- Acute sinusitis should be treated with decon-
gestants and antibiotics for 10-14 days
- If chronic sinusitis is suspected predisposing
factors-such as allergy, deviated nasal septum,
and intranasal polyps-should be treated first
- Chronic sinusitis should be treated with
broad spectrum antibiotics, with topical steroids
and antihistamines if indicated; if treatment fails
then computed tomography should be done,
followed by surgery if indicated
- Fungal and dental sinusitis should be con-
sidered
- Obstruction of nasal airway by hypertrophy
of tonsils and adenoids may cause sinusitis in
children
- Elderly patients must be treated aggressively
to prevent exacerbation of chest disease
- Immunocompromised patients with facial
pain, headache, or fever of unknown origin
should receive early, aggressive treatment

cilia and is moved constantly along predetermined pathways to the sinus ostia. In the frontal sinus mucus passes up along the intersinus septum, then across the roof of the sinus before returning across the sinus floor to the frontal recess and the middle meatus. ${ }^{6}$ In the maxillary sinus mucus is moved from the floor of the sinus radially and up the walls of the sinus to the superiorly placed ostium (fig 1 ).

Sinusitis occurs when this transport mechanism fails, usually in response to a virus, bacterium, or allergen. Less common causes of failure include fungi, mechanical obstruction, trauma, the mucosal oedema associated with pregnancy, and primary ciliary dysfunction such as Kartagener and Young's syndromes (fig 2 and box).?

Mucociliary transport is impaired by the hypersecretion of mucus and inflammatory mediators released in response to infection or allergy. ${ }^{8}$ The trapped mediators cause mucosal oedema leading to further loss of ciliary function, obstruction of the ostia, congestion, pain, and sensation of pressure. ${ }^{69-11}$ Ostial obstruction leads to intrasinus hypoxia after the absorption of oxygen by the vascular mucosa. The resulting increase in acidity leads to further mucociliary dysfunction. Thirty per cent of the ciliated cells may undergo metaplastic change to mucus secreting 


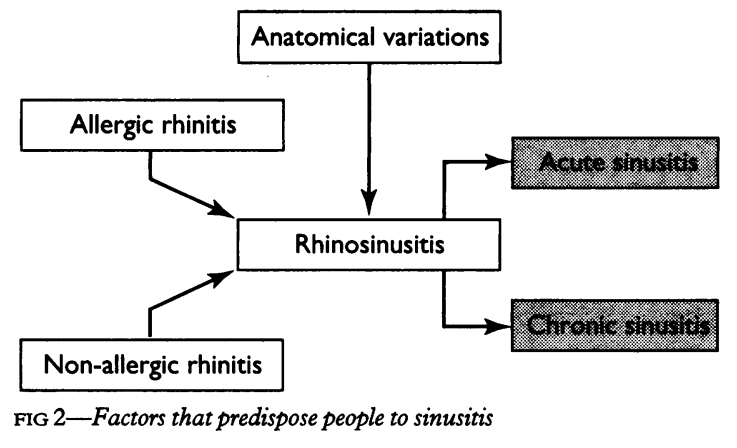

\author{
Causes of non-allergic rhinitis \\ - Vasomotor disturbances \\ - Mucociliary dysfunction: \\ Primary-Kartagener's syndrome \\ Secondary-infection \\ - Immune deficiency \\ - Hormonal factors: \\ Puberty \\ Pregnancy \\ Oral contraception \\ Senile rhinorrhoea \\ - Iatrogenic factors \\ Rhinitis medicamentosa \\ Misuse of cocaine \\ Aggressive surgery
}

goblet cells, and structural damage to the epithelium has been correlated with a decrease in ciliary beat frequency from the normal 700 beats per minute to less than $300 .{ }^{1213}$ As the disease progresses the infected mucous membrane balloons, and the epithelium becomes irreversibly damaged with formation of

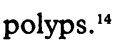

Poor sinonasal drainage and retention of secretions also occur secondarily to apposition of mucociliary surfaces. ${ }^{6}$ The ethmoid infundibulum and middle meatus are the areas most commonly affected by anatomical variation leading to such apposition. The release of the neuropeptide substance $P$, which controls pain perception in the nasal mucosa, contributes to headache and pain. Substance $P$ stimulates the unmyelinated $\mathrm{C}$ fibres leading to extravasation of fluid, vasodilatation, and hypersecretion. ${ }^{14}$ The decrease in ostial diameter and increase in nasal airway resistance, with a change in posture, also contributes to pain. ${ }^{15}$

\section{Microbiology}

Seventy per cent of acute sinusitis is caused by Streptococcus pneumoniae and Haemophilus influenzae, of which a proportion produce $\beta$ lactamase. ${ }^{8}$ Branhamella catarrhalis is a common cause of acute sinusitis in children. Staphylococcus aureus is identified in nasal cultures of $30 \%$ of the asymptomatic population but rarely causes acute sinusitis. Nasal swabs are therefore of limited value because the mixed nasal flora does not usually represent the sinus pathogen. ${ }^{8}$ Over 200 viruses have been implicated; most act by disorganising the cilial movement, while others rapidly replicate in the cell, leading to cell death. ${ }^{8}$

The pathogens in chronic sinusitis are often opportunistic and include anaerobes-such as $\alpha$ haemolytic streptococci, bacteroides, veillonella, and corynebacterium ${ }^{16}-H$ influenzae in $60 \%$ of cases, ${ }^{8}$ and $S$ aureus. Pseudomonas aeruginosa is common in patients with nasal polyps or cystic fibrosis. Culture of sinus aspirate may not produce growth unless strict anaerobic techniques are used. Fungi are normal commensals of the upper airway but occasionally lead to chronic sinusitis, especially in diabetic or immunocompromised patients.

\section{Symptoms}

Symptoms of acute sinusitis include preceding upper respiratory tract infection, mucopurulent rhinorrhoea, postnasal drip, cough, fever, nausea, and congestion. Facial pain above or below the eyes that increases on leaning forwards is common. Few symptoms are highly specific except for toothache, but this occurs in only $11 \%$ of patients. ${ }^{17}$ The site of pain or headache may implicate a specific sinus (fig 3 ).

The symptoms of chronic sinusitis are similar to those of acute sinusitis but often less specific. A patient may complain only of a chronic, unproductive cough, persistent laryngitis, or a foul taste. Mucopurulent rhinorrhoea presents frequently with periodic acute exacerbations. Fifty per cent of patients present with severe headache, which may be maximal three to four hours after getting up in the morning and then slowly improves. ${ }^{14}$ More detailed evaluation is needed if symptoms occur three to four times a year, are not controlled by steam inhalation and non-prescription medicines, and affect more than one anatomical site or if the sinusitis is associated with exacerbation of asthma. ${ }^{18}$

\section{Signs}

The nasal airway is often reduced and the mucosa hyperaemic with purulent secretions drying to form crusts. Prolonged inflammation leads to polypoid change, especially of the middle meatus and middle turbinate. Nasal allergy may be indicated by pale oedematous mucosa and nasal polyps. ${ }^{4}$

The view of the nasal cavity with a thudicium speculum is limited, even after the application of topical decongestants. Factors predisposing to sinusitis, such as deviation of the nasal septum, septal spurs, hypertrophic turbinates, and nasal polyps, are identified. Pus in the middle meatus indicates frontal, anterior ethmoid, or maxillary sinusitis, but absence of pus does not preclude the diagnosis. Rhinoscopy with a flexible or rigid endoscope allows more detailed examination. A rigid endoscope has the advantage of better optical definition and can be introduced into the middle meatus, allowing anatomical abnormalities to be identified.

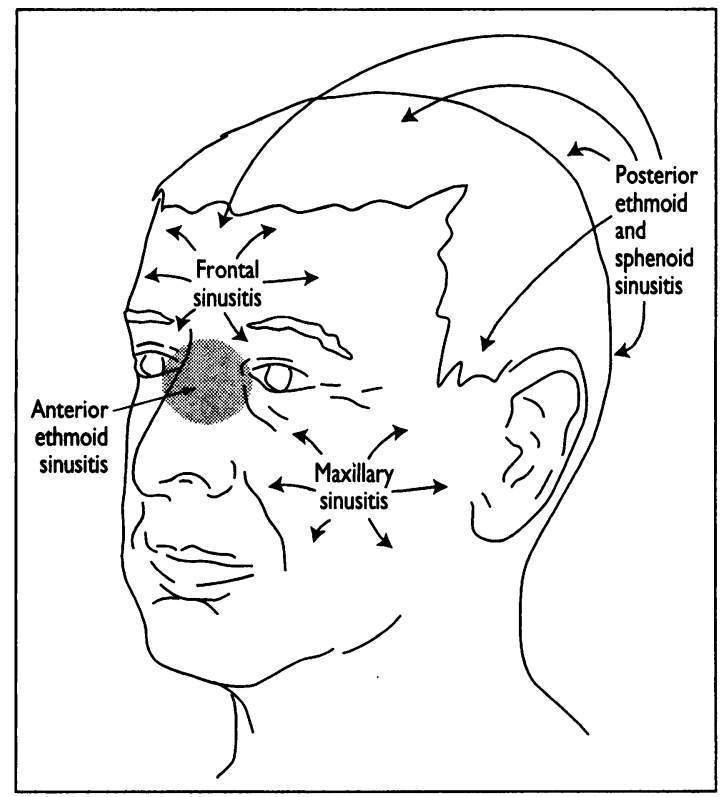

FIG 3-Sites of referred pain from individual sinuses 


\section{Investigations}

Comparative studies of nasal cytology and plain $x$ ray films of sinuses have shown a $79 \%$ correlation, although the authors concluded that nasal cytology should not be considered to be an adequate alternative to plain $x$ ray examination. ${ }^{19} 20$ Specific allergens may be identified by skin prick testing, which is rapid and cheap, and a variety of allergens can be tested. It carries a small risk, however, of anaphylaxis. An alternative test is the radioallergosorbent test, which is more time consuming but provides quantitative information about specific sensitivities. Ultrasound examination of the sinuses does not need ionising radiation, but its reliability in identifying fluid with a positive echo in the A mode when compared with sinus aspiration is disputed. $20-22$

Transillumination requires a completely darkened room. A light is shone through the maxillary or frontal sinus, and the amount of light transmitted through the palate is observed, or vice versa. Disadvantages of this technique include poor correlation with the presence of fluid, as proved by antral washout, ${ }^{23}$ poor consistency among observers, and the inability to evaluate the ethmoid and sphenoid sinuses. A limited place remains for its use in patients who resist ionising radiation or who are pregnant. A positive result can be assumed only when gross differences in sinus aeration occur on each side.

\section{RADIOLOGY}

\section{Plain $x$ ray examination}

Three views commonly taken in plain $x$ ray examinations are an occipitomental (Waters), an occipitofrontal (Caldwell), and a lateral view to demonstrate the sphenoid sinus. Some departments offer only the occipitomental view as the other views yield little additional information. The place of such films in investigating uncomplicated acute sinusitis is debatable as the findings of air fluid levels or opacity rarely lead to a change in management (fig 4). There is further debate on whether doctors in primary health care, as well as specialists, should be allowed to request routine $x$ ray examinations of sinuses. ${ }^{24}$

In chronic sinusitis the findings are less clear. Air fluid levels are unusual, and a more common finding is mucosal thickening or sinus opacity. The ethmoid sinuses are poorly visualised, and poor correlation exists between the extent of abnormality of the sinus and the severity of the symptoms. ${ }^{25}{ }^{26}$ Interpretation of $x$ ray films is subject to variation among observers. Some studies have suggested that mucosal thickening of 6-8 $\mathrm{mm}$ in $x$ ray pictures is of clinical importance..$^{82}$ A correlation between the degree of opacification and antral puncture has been shown, ${ }^{28}$ but abnormal radiological findings may also be found in asymptomatic patients-for example, in up to $50 \%$ of well children. ${ }^{29}$

\section{Computed tomography}

Coronal computed tomography is currently the gold standard of radiographic evaluation of chronic inflam-

FIG 4-Plain $x$ ray film showing air-fluid level in right maxillary antrum and mucosal thickening in left maxillary antrum. Reproduced from

Shankar et al ${ }^{30}$ with permission

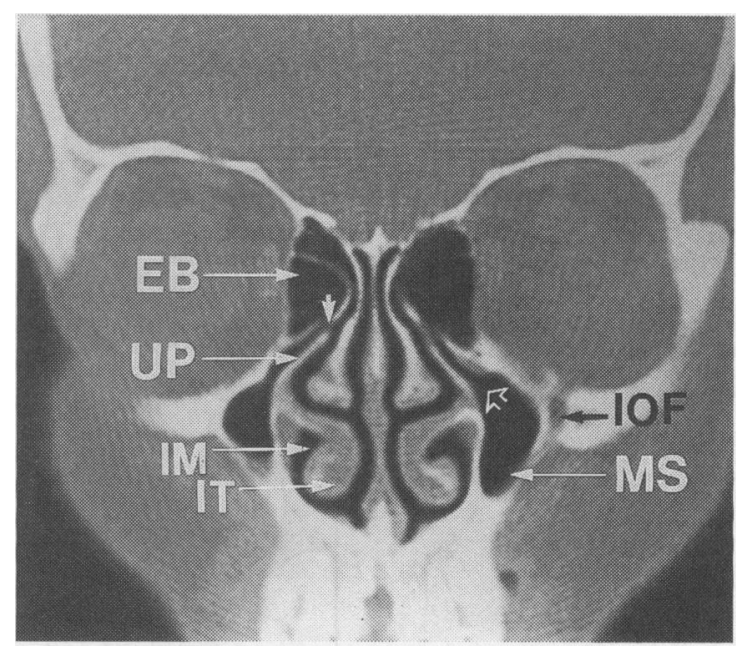

FIG 5-Computed tomogram showing normal maxillary sinus. The anterior portion of the maxillary sinus (MS) drains through the maxillary ostium into the ethmoid infundibulum (open arrow), which is bounded superolaterally by the ethmoid bulla (EB) and inferomedially by the uncinate process (UP). The ethmoid infundibulum opens through the hiatus semilunaris, which is a two dimensional slitlike opening that connects the infundibulum with the middle meatus (short, white arrow). The infraorbital foramen (IOF), inferior turbinate (IT), and inferior meatus (IM) are also shown. Reproduced from Shankar et al ${ }^{30}$ with permisison

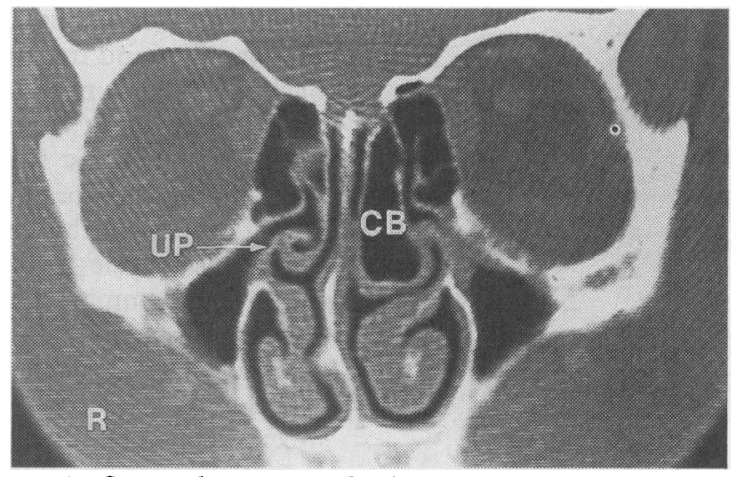

FIG 6-Computed tomogram showing anatomical variations that reduce ventilation of the ostiomeatal complex, predisposing patients to acute and chronic sinusitis. These variations would not be identified in plain $x$ ray films. Reproduced from Shankar et al ${ }^{\circ}$ with permission

mation of the paranasal sinuses. ${ }^{30}$ It is not economically possible or medically necessary to scan every patient presenting with symptoms suggestive of chronic sinusitis. Computed tomography should be requested only after failure of maximal medical treatment, if a complication arises, or if malignancy is suspected. ${ }^{4}$

Computed tomography not only identifies soft tissue abnormalities but provides a surgical map of the paranasal sinuses (fig 5). The level of the cribriform plate, bony dehiscence of the lamina papyracea or optic canal, and the relation of the optic nerve to the posterior ethmoid sinus are identified preoperatively. Anatomical variations are identified which would not be seen on endoscopic examination of the nose but which may compromise the ostiomeatal complexfor example, pneumatisation of the middle turbinate (fig 6) or Haller cells in the inferomedial borders of the orbit. Sixty two per cent of the population with chronic sinusitis have at least one anatomical variation compared with $11 \%$ of a normal control group (fig 7). ${ }^{31}$

Computed tomography has permitted a more widespread acceptance of functional endoscopic sinus surgery, which has radically changed the surgical approach to inflammatory sinus disease. ${ }^{32}$ It is useful for showing patients the location of disease and the surgical approach, but computed tomography should not be the sole basis for operating, especially as $24-39 \%$ of the asymptomatic population may demonstrate mucosal disease. ${ }^{33}$ 
FIG 7-Most common anatomical variations that predispose people to chronic sinusitis

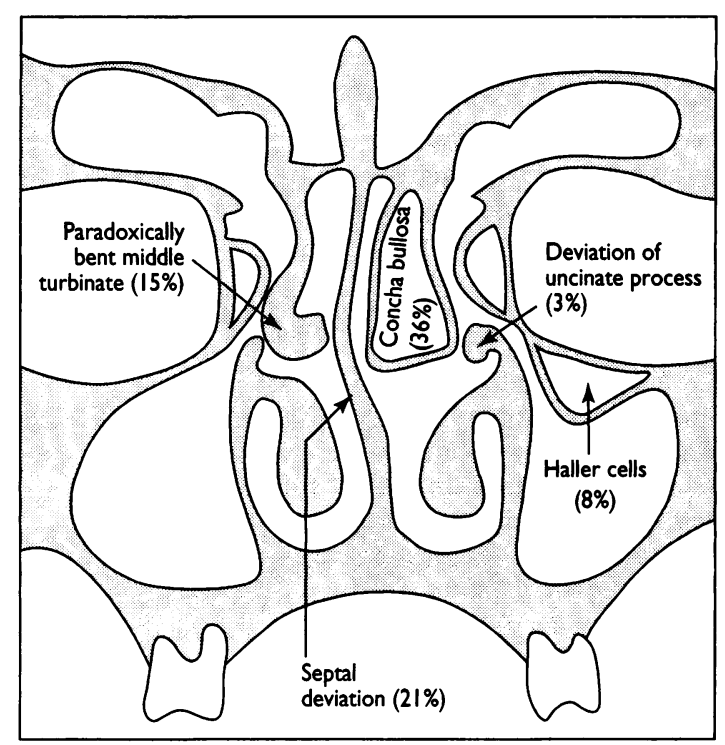

Magnetic resonance imaging

Magnetic resonance imaging provides high definition scans of sections of soft tissue without ionising radiation, but it cannot demonstrate bone. Its role in sinus disease has been reviewed. ${ }^{3034}$ The mucosa of the ethmoid sinuses and nasal cavity have a natural cycle of vasodilatation and mucosal oedema followed by vasoconstriction and mucosal shrinkage, and magnetic resonance imaging is therefore of limited value in investigating chronic sinusitis, which is secondary to disease of the anterior ethmoids. The signal intensity of normal mucosa in this region in its oedematous phase of the nasal cycle is indistinguishable from that of extensive inflammatory disease. ${ }^{35}$ The frontal, maxillary, and sphenoid sinuses do not have such a physiological cycle, and magnetic resonance imaging can be helpful.

Certain disease processes may be differentiated by magnetic resonance imaging. Inflammatory conditions exhibit high signal intensity on $\mathrm{T} 2$ weighted images, whereas neoplastic processes, of which $90 \%$ are squamous cell carcinoma, assume an intermediate bright signal on T2 weighted images. ${ }^{36}$ Fungal concretions have low signal intensities on $\mathrm{T} 2$ weighted images, similar to air. ${ }^{37}$ As with computed tomography, a notable rate of false positive results occurs.

\section{Treatment}

The aims of treatment of sinusitis are eradication of infection, reducing inflammation and the restoration of ventilation of the sinuses allowing the return of normal mucociliary function. ${ }^{37}$ If caught early one may even interrupt the cycle which leads to polyp formation. The box outlines treatment for both chronic and acute sinusitis.

\section{MEDICAL TREATMENT}

Acute sinusitis

Acute sinusitis requires treatment with antibiotics for an adequate length of time, usually two weeks. Several antibiotics are effective against the commonly encountered pathogens, and their efficacy has been compared by repeated antral puncture and culture of sinus aspirate. These antibiotics include amoxycillin with and without clavulanic acid, trimethoprim with sulphamethoxazole, cefuroxime, erythromycin, and azithromycin. Differences in efficacy against bacteria and anaerobes that produce $\beta$ lactamase exist, depending on the antibiotic. Amoxycillin fails in the $20-30 \%$ of cases of $H$ influenzae that produce $\beta$ lactamase, whereas augmented penicillin, trimethoprim with sulpha- methoxazole, and cefuroxime axetil do not. Minor differences occur in patients' tolerance of these antibiotics with regard to gastrointestinal upset. ${ }^{389}$ Anaerobes isolated in sinusitis are typically sensitive to penicillin, but complications involving the orbit or intracranial cavity need parenteral broad cover. ${ }^{8}$

The effect of topical steroids on acute sinusitis is modest. They may be of value in reducing facial pain more quickly. ${ }^{40}$

Decongestants can reduce local oedema, promoting drainage and ventilation. Topical decongestants vary in duration of action. Xylometazoline is a long acting decongestant with the disadvantage of causing rebound vasodilatation if used for more than five days; overuse results in rhinitis medicamentosa. Xylometazoline is not recommended for the long term treatment of sinusitis. Topical nasal decongestants are more effective if given with the patient in the "Mecca position," which should be maintained for two to three minutes (fig 8). ${ }^{41}$ Systemic decongestants may provide relief from symptoms but have adverse side effects secondary to $\alpha$ adrenergic stimulation, such as nervousness, insomnia, tachycardia, and hypertension.

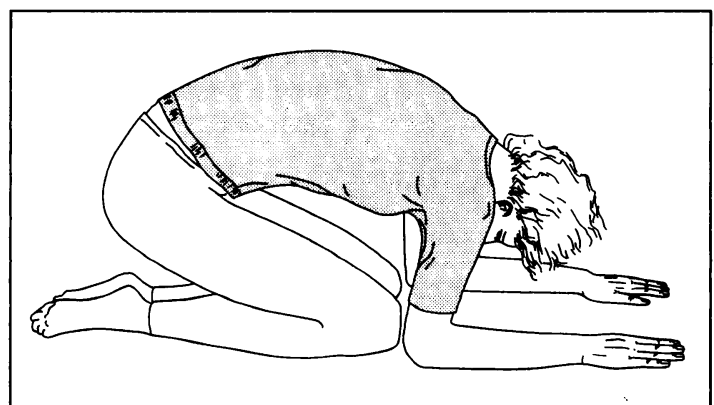

FIG 8- "Mecca position" for receiving topical nasal decongestants

Mucolytics such as guaiphenesin, although difficult to assess objectively, may improve nasal mucociliary clearance by improving the viscoelastic properties of the secretions, thus making them easier to clear. Penetration by antibiotics and topical medicines may also be enhanced. ${ }^{42}$ Nasal toilet with steam inhalation and alkaline or saline douching may also lead to relief from symptoms by softening crusts and moisturising dry mucosa.

\section{Chronic sinusitis}

Most recurrent or chronic sinusitis is secondary to disease in the anterior ethmoid complex, and early identification of predisposing factors is the key to successful treatment. Infection secondary to patho-

\section{Treatment plan for acute and chronic} sinusitis

\section{Acute sinusitis}

Analgesia

Antibiotics for 10-14 days

Topical decongestants

Steam

Antral washout

Urgent treatment of intraorbital and intracranial complications

\section{Chronic sinusitis}

Identification of predisposing factors and treatment of these with topical steroids or long course of antibiotics

Alkaline nasal douche, with or without steam

If no improvement, coronal computed tomography

Functional endoscopic sinus surgery if indicated; continued medical treatment with antibiotics and topical steroids as appropriate postoperatively 
physiological abnormalities requires medical treatment including antibiotics, topical steroids, antihistamines, and mast cell stabilisers. Chronic sinusitis secondary to anatomical abnormality can benefit from precise surgical intervention. Most cases have a combination of pathophysiological and anatomical factors which predispose patients to chronic infection, and medical treatment both precedes surgery and continues after surgery when conditions, such as allergy, persist.

Initial treatment comprises a combination of antibiotics, topical steroids, and antihistamines. Antibiotics similar to those used in acute sinusitis are advocated for prolonged courses of between two and six weeks. Consideration must be given to the production of $\beta$ lactamase or the presence of anaerobes. Decongestants have a role as previously described, and simple inhalation of steam helps with nasal toilet and promotes clearance of secretions while providing relief from symptoms.

Topical corticosteroids suppress the inflammatory response and, by reducing mucosal oedema in the ostiomeatal complex, promote drainage and ventilation of the sinuses. ${ }^{3}$ To be effective they must reach the affected mucosa so obstruction of the airway by hypertrophy of the turbinates, septal deviation, or polyps must be corrected. Recommended doses must be used for at least two weeks until the maximal effect is noted. All topical corticosteroids cause a degree of nasal irritation, crusting, and bleeding. Systematic effects are not noted with overuse until 4-16 times the recommended dose, depending on the preparation, is given..$^{43}$

Antihistamines compete for $\mathrm{H}_{1}$ binding sites on target cells in respiratory mucosa. The newer second generation antihistamines do not cross the blood-brain barrier and are less sedative than the first generation drugs. Excess dryness in the nose, crusting, and tachyphylaxis have not been observed with the nonsedating antihistamines. Mast cell stabilisers such as topical sodium cromoglycate prevent degranulation of mast cells in allergic rhinitis and can both prevent and treat an allergic reaction.

\section{SURGICAL TREATMENT}

Acute sinusitis

Most acute sinusitis responds to medical treatment, but persistent symptoms may require antral washout either for a diagnostic aspirate or for providing relief from symptoms. Traditionally antral washouts are conducted through the inferior meatus, but the sublabial route through the canine fossa can also be used. Culture of mucopus is important in disease that is resistant to medical treatment, and it is regarded as essential in cystic fibrosis or nosocomial infection.

\section{Chronic sinusitis}

Surgery is considered if chronic sinusitis fails to respond to adequate medical treatment, complications are likely, or an anatomical abnormality is identified that makes recurrent acute sinusitis more likely. Despite the ethmoid sinus having been identified as the origin of chronic sinusitis as early as the 19th century surgery focused on the maxillary sinus until the mid1980 s. Both inferior meatal antrostomy and the radical sublabial antrostomy described by Caldwell and Luc have been popular in the management of chronic sinusitis. An inferior meatal antrostomy is placed laterally to the inferior turbinate and level with the floor of the nose allowing drainage of the maxillary sinus when excess secretions accumulate; in less overwhelming circumstances the cilia continue to transport mucus to the natural ostium. ${ }^{44}$ The natural ostium remains obstructed and the secretions continue to accumulate. A retrospective trial found that $45 \%$ of inferior meatal antrostomies closed spontaneously; closure was more likely if the diameter was less than $1 \mathrm{~cm}$ and in children aged under $16 . .^{45}$

The work of Messerklinger ${ }^{46}$ and Stammberger ${ }^{44}{ }^{77}$ and the advent of Hopkins rods and computed tomography have revolutionised the surgical approach to chronic sinusitis. Functional endoscopic sinus surgery specifically aims at restoring the physiological function of the sinuses, and has been quoted as having success rates of $80-95 \%{ }^{48}{ }^{49}$ Recurrence of disease after endoscopic sinus surgery is usually persistent disease that has begun to show symptoms. ${ }^{50}$ The rigid endoscope allows better diagnostic assessment of the nasal cavity and close monitoring of postoperative progress. Endoscopic intranasal surgery now includes dacryocystorhinostomy, drainage of mucoceles, management of cerebrospinal fluid rhinorrhoea, orbital decompression, and decompression of the optic nerve after traumatic entrapment.

The operating conditions for functional endoscopic sinus surgery may be better with local anaesthesia and sedation than with general anaesthesia because bleeding and venous congestion are reduced. The orbit and skull base are sensitive, and an awake patient can warn the surgeon if he or she is close to a danger area. ${ }^{50}$

Anatomical abnormality or soft tissue abnormality in the ostiomeatal complex (fig 9) that is identified preoperatively is resected to help ventilate the sinuses and promote clearance of mucus from the frontal and maxillary sinuses into the middle meatus (fig 10).49 Great care must be taken around the frontal recess as scarring may lead to persistent frontal sinus disease or formation of mucoceles. ${ }^{50}$ If indicated, the posterior ethmoid may be entered through the ground lamella and dissection continued back to the sphenoid. Stammberger advocates dissection of the ethmoid sinuses from anterior to posterior whereas Wigand advocates a posterior to anterior sphenoethmoidectomy. ${ }^{51}$

After surgery for chronic sinusitis it may take many

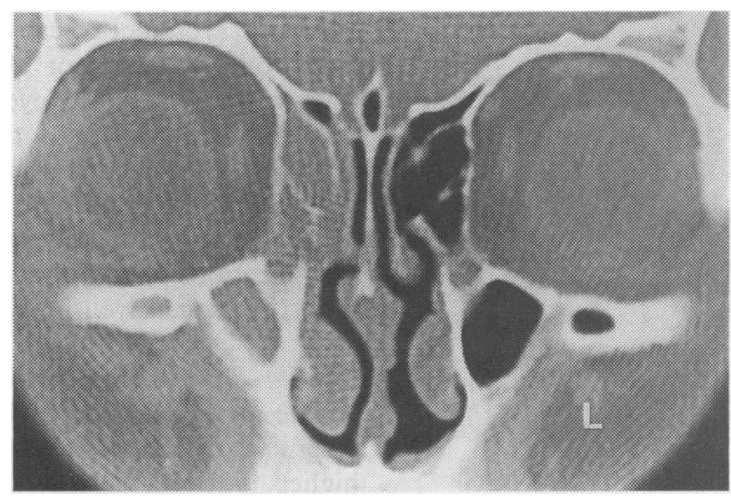

FIG 9-Computed tomogram showing unilateral, right sided pansinusitis, with inflammatory disease extending into frontal recess. Reproduced from Shankar et a ${ }^{30}$ with permission

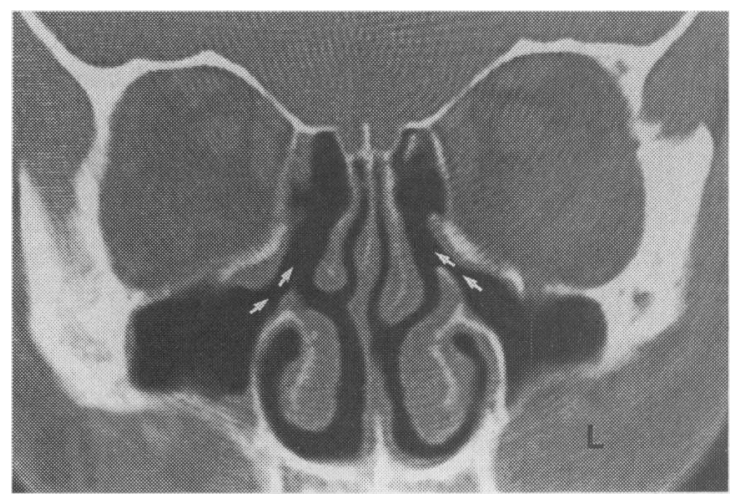

FIG 10-Computed tomogram after functional endoscopic sinus surgery showing complete bilateral anterior ethmoidectomy. The ostiomeatal complexes are patent (white arrows). The uncinate process has been completely removed on the right and partially resected on the left. Reproduced from Shankar et a ${ }^{30}$ with permission 
months for mucosal changes to reverse. Alkaline or saline nasal douching with or without steaming should be conducted-ideally, twice daily. Antibiotics may be needed for several weeks until the postnasal drip stops. Topical steroids and antihistamines may also be required, for several months or long term.

Functional endoscopic sinus surgery is not without risk, and complications include breach of the lamina papyracea, with diplopia, intraorbital haemorrhage, or infection; blindness after transection of the optic nerve; leak of cerebrospinal fluid, usually through the lateral lamella of the cribriform plate; and injury to the internal carotid artery. The quoted complication rates vary between $8 \%$ and $0.3 \% .{ }^{52}$

Endoscopic intranasal surgery is contraindicated in the presence of osteomyelitis, inaccessible lateral frontal sinus disease, stenosis of the frontonasal duct, and threatened intraorbital or intracranial infection, as well as in the absence of defined abnormalities of the ostiomeatal complex. ${ }^{50}$ In such cases the external approach to the ethmoid sinuses allows safe identification of the skull base and orbit. Limitations of this approach include poor access to the frontal recess and the most anterior ethmoid air cells and a facial scar.

\section{Special considerations}

\section{COMPLICATIONS}

The most worrying complications of sinusitis involve the orbit and intracranial cavity. Before the introduction of antibiotics cellulitis affecting the orbit secondary to sinusitis led to blindness in $20 \%$ of patients and death from meningitis or intracranial abscess in $17 \% .{ }^{53}$ Even today visual loss in $10 \%$ of patients has been described in the literature..$^{54}$ Orbital cellulitis is divided into two groups by its relation with the orbital septum. Disease anterior to the orbital septum occurs more commonly, and it rarely threatens vision; disease posterior to the orbital septum is related either to a subperiosteal collection of pus or to an intraorbital cellulitis or abscess. This second group causes proptosis of the eyeball, and the risk of loss of sight is high. These patients need to be assessed urgently with axial computed tomography and given intravenous broad spectrum antibiotics, and, if indicated, have urgent surgical drainage. Other complications include the development of mucoceles (fig 11) or osteomyelitis.

\section{FUNGAL SINUSITIS}

The increase in the incidence of fungal sinusitis in recent years is a result of better diagnostic facilities, a higher index of suspicion, and an increase in predisposing causes such as radiotherapy, chemotherapy, immunosuppressive therapy, and immunodeficiency diseases such as AIDS. Fungal sinusitis has four distinct clinicopathological syndromes, each with its own histopathological features.

Indolent fungal sinusitis is characterised by indolent

FIG 11 -Computed tomogram showing mucoceles affecting bilateral concha bullosa with extensive disease of ostiomeatal complex. In plain films this would show as mucosal thickening. Reproduced from Shankar et al with permission

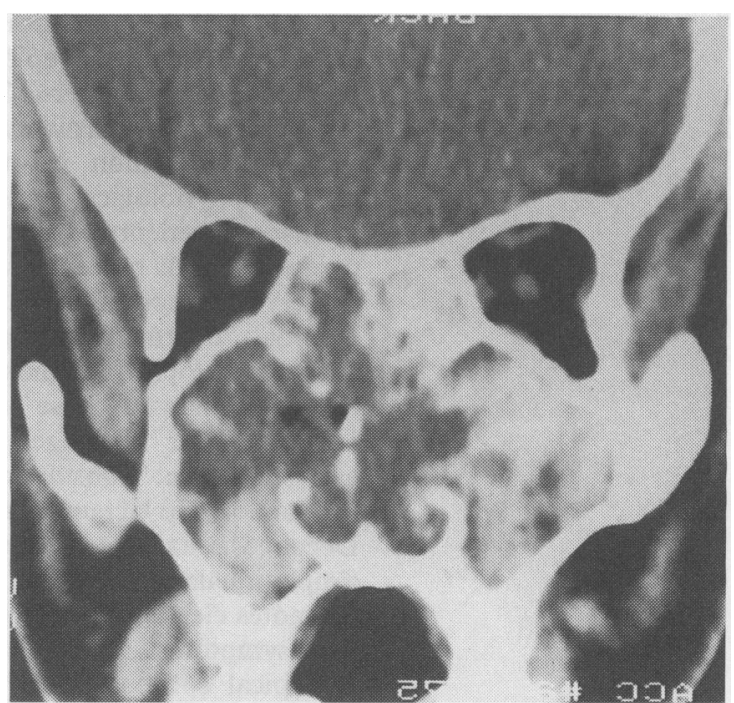

FIG 12-Computed tomogram showing inflammatory reaction throughout sinuses related to fungal (aspergillus) sinusitis. The high density areas are the result of accumulation of heavy metals in the mycelia. Reproduced from Shankar et al ${ }^{\beta 0}$ with permission

granulomas with giant cells. The clinical features are of chronic sinusitis and may include a mouldy smell or substantial nasal crusting. The colour of the infected matter ranges from whitish yellow to dirty brown or grey-black and is buttery in consistency. Increasing pressure and inflammation may lead to local bony destruction. Computed tomography shows dense radio-opaque masses in the sinus (fig 12).

Mycetoma is a mass of hyphae on the mucous membrane that supplies nutrients. It can be removed endoscopically, but if dense a formal Caldwell-Luc procedure is indicated..$^{55}$

Invasive fungal sinusitis is characterised by soft tissue necrosis, fibrosis, and fungal invasion of vessels, leading to orbital complications, meningitis, and involvement of the central nervous system. It usually occurs in immunocompromised patients and needs aggressive medical treatment and wide debridement.

Allergic fungal sinusitis has similar features to allergic bronchopulmonary aspergillosis. ${ }^{56}$ This non-invasive condition occurs in atopic patients, $75 \%$ of whom have asthma and $85 \%$ nasal polyps. The condition is resistant to conventional treatment and affects multiple sinuses. Sinus aspirate is usually of a similar consistency to peanut butter and has the characteristics of allergic mucin, containing eosinophils, CharcotLeyden crystals and fungal hyphae. Treatment is drainage and ventilation. Steroids decrease the inflammatory response as in allergic bronchopulmonary aspergillosis. $^{56}$

\section{DENTAL SINUSITIS}

Odontogenic sinusitis may be related to periapical or periodontal disease, as well as to oroantral fistula. The latter usually occurs after extraction of teeth, particularly molar teeth. The risk is greater in adults than in children because of the continued expansion of the sinus into the alveolar bone after the eruption of the secondary dentition. ${ }^{57}$

Symptoms of dental sinusitis are usually facial pain, swelling, tenderness, and discharge from the nose or an oral fistula. The maxillary sinus is often opaque in a plain $x$ ray film..$^{57}$ Anaerobic organisms predominate, and antibiotics should be chosen appropriately. The dental disease needs to be eradicated, otherwise the sinusitis will persist.

\section{PAEDIATRIC SINUSITIS}

Awareness is increasing of chronic sinus disease in children. Predisposing factors include hypertrophy or recurrent infection of the tonsils and adenoids, allergy, 
and cleft palate. These factors should be addressed before sinus surgery is considered. The causal relation between the adenoids and sinusitis is unclear, but enlarged adenoids cause stasis of secretions and secondary inflammation of the sinus ostia. Removal of non-obstructive adenoids has little benefit. Symptoms of sinus disease in children include rhinorrhoea, cough, fetor, headache, and low grade fever..$^{58}$

\section{Common sites and bacteria}

Computed tomography confirms that the commonest sites of disease are the ethmoid infundibulum and the anterior ethmoid complex. The commonest bacteria are Streptococcus pneumoniae, Haemophilus influenzae, and Branhamella catarrhalis. ${ }^{585}$ Culture of ethmoid biopsy specimens in patients unresponsive to medical treatment found $B$ catarrhalis, most of which produced $\beta$ lactamase, to be common in children aged under 4 years and Staphylococcus aureus to be common in children aged over $4 .^{58} S$ aureus and anaerobes were more common in those whose symptoms had persisted for more than one year. For these reasons Augmentin and cefuroxime are of value in the management of chronic sinusitis in children. ${ }^{58}$

Surgery is indicated if medical treatment fails. Antral washout requires extra care if the secondary dentition has not erupted. As with adults, endoscopic intranasal procedures have become more popular, but nasal endoscopy in outpatient clinics is not well tolerated by children, and endoscopic surgery is considered after evaluation by computed tomography. Paediatric endoscopic surgery is always conducted under general anaesthesia after a topical decongestant has been tried. Occasionally a limited septoplasty or reduction of the middle turbinate is needed to allow access to the middle meatus. Endoscopic surgery in children is similar to that in adults, although the confined space naturally increases the surgical risks. Postoperative medical treatment is similar to that in adults except that crusts and granulation tissue are removed under a second general anaesthetic two to three weeks after surgery. Eighty per cent of children with both asthma and sinusitis improve after their sinusitis is controlled, and failure of surgery is associated with immunodeficiency, cystic fibrosis, the immotile cilia syndrome, and chronicity of disease..$^{59}$

\section{ELDERLY PEOPLE}

People aged over 60 constitute an increasing proportion of the general population. If neglected, sinusitis exacerbates chest disease, which is more threatening in this age group. The clinical features, bacteriology, and investigation of this group are closely similar to those of the general population, but complications occur more readily and need aggressive treatment. Early medical intervention is important to reduce morbidity. ${ }^{60}$

\section{CRITICALLY ILL PATIENTS}

Sinusitis is an important cause of sepsis in critically ill patients and may be difficult to identify because of the many other sites that may become infected. Risk factors include craniofacial injury (haematoma in the sinuses may become secondarily infected), prolonged

\section{Sinusitis in immunocompromised patients}

- Need for high index of suspicion, especially if headache, facial pain, fever of unknown origin, or nasal crusting is presen

- Urgent broad spectrum parenteral antibiotic

- Urgent computed tomography

- Surgical drainage and debridement nasogastric or nasotracheal intubation, and the prescription of steroids. ${ }^{61}$ The incidence of sinusitis has been quoted as $26 \%$ in the patients in intensive care units who require nasotracheal intubation. ${ }^{61}$ The diagnosis may be delayed because the clinical signs are limited, and transfer for computed tomography is not without risks. Bedside diagnostic antral aspiration is considered to have a lower morbidity. Precautionary measures such as saline nasal douching and topical decongestant have not been shown to be beneficial. ${ }^{61}$

\section{Immunocompromised patients}

Opportunistic infections occur in immunosuppressed patients, often in those with neoplastic disease such as lymphoproliferative disease, especially leukaemia. Severe fungal infections were found at necropsy in $22-28 \%$ of patients with leukaemia. ${ }^{62}$ Prolonged granulocytopenia makes invasive fungal infection more likely to occur in the lungs, but severe disease of the sinuses has been reported. ${ }^{63}$ The fungal species include Aspergillus, Candida, Rhizopus, and Mucor.

Sinusitis must be considered in an immunosuppressed patient presenting with fever, facial pain, swelling, and nasal crusting or ulceration. $x$ Ray films may show only slight mucosal thickening, ${ }^{63}$ but if this is abnormal then a regimen of antibacterial and antifungal agents should be started immediately. Surgical biopsy for culture and drainage should be undertaken when appropriate (box).

Patients who are HIV positive have deficits in both cell mediated and humoral immunity and are more susceptible to bacterial infection. Sinusitis occurs in $75 \%$ of those with AIDS and is often extensive and difficult to treat, especially if the CD4 count is $<200 \times 10^{\circ} / 1 .{ }^{64}$ As is the case in patients who are HIV negative, the ethmoid and maxillary sinuses are predominantly involved. Failure to respond to medical treatment is an indication for antral aspiration to allow bacteriological identification and to indicate appropriate antibiotic treatment. ${ }^{65}$ Pathogens include Cryptococcus neoformans, cytomegalovirus, and Pseudomonas aeruginosa. This last can cause a type of osteomyelitis that is similar to necrotising otitis externa.

A patient who is HIV positive presenting with headache requires early investigation to exclude meningitis, and procedures should include computed tomography or magnetic resonance imaging, or both, together with lumbar puncture. If the infection is uncomplicated a 10 day course of doxycycline may suffice. ${ }^{65}$ If a patient is systematically unwell he or she should be admitted for antral washout and intravenous antibiotics. Failure to respond may be an indication for urgent surgery.

1 US Department of Health and Human Services. NIH data book. Bethesda, MD: DHHS, 1984:57.

2 Department of Health and Social Security. Digest of health statistics. London: HMSO, 1971.

Slavin RG. Sinusitis in adults. 7 Allergy Clin Immunol 1988;81:1028-32.

4 Calhoun K. Diagnosis and management of sinusitis in the allergic patient. Otolaryngol Head Neck Surg 1992;107:850-4.

5 Stammberger H. Functional endoscopic sinus surgery. Philadelphia: Decker, 1991.

6 Messerklinger W. On the drainage of the normal frontal sinus of man. Acto Otolaryngol 1967;643:176-81.

7 Mackay IS. Rhinitis and sinusitis. British foumal of Diseases of the Chest 1988;82:1-8.

8 Yonkers AJ. Sinusitis-inspecting the causes and treatment. Ear, Nose and Throat fournal 1992;71:258-62.

9 Wilson R, Sykes DA, Currie DC, Cole PJ. Beat frequency of cilia from sites of purulent infection. Thorax 1986;41:453-8.

10 Slavin RG, Zilliox AP, Samuels LD. Is there such an entity as allergic sinusitis? [Abstract] $f$ Allergy Clin Immunol 1988;81:284.

11 Reilly JS. The sinusitis cycle. Otolaryngol Head Neck Surg 1990;103(suppl): 856-62.

12 Ohasi Y, Nakai Y. Reduced ciliary activity in chronic sinusitis. Acto Otolaryngol 1983;397(suppl):3-9.

13 Ohasi Y, Nakai Y. Functional and morphological pathology of chronic sinusitis mucous membrane. Acta Otolaryngol 1983;397:11-48.

14 Stammberger H, Wolf G. Headaches and sinus disease: the endoscopic approach. Ann Otol Rhinol Laryngol 1988;134(suppl):3-23.

15 Melen I, Friberg BO, Andreasson L, Ivarsson A, Jannert M, Lindahl L. Effects of phenolpropanolamine on ostial patency in patients treated for chronic maxillary sinusitis. Acta Otolaryngol (Stockh) 1986;101:494-500. 16 Winther B, Gwaltney JM Jr. Therapeutic approach to sinusitis: antiinfectious 
therapy as the baseline of management. Orolaryngol Head Neck Surg 1990;103(suppl):876-9.

17 Williams JW, Simel DL, Roberts L, Samsa GP. Clinical evaluation of sinusitis-making the diagnosis by history and physical examination. $A n n$ Intern Med 1992;117:705-10.

18 Druce HM. Diagnosis of sinusitis in adults: history, physical examination, nasal cytology, echo, and rhinoscope. I Allergy Clin Immunol 1992;90: 436-41.

19 Wilson NW, Jalowayski AA, Hamburger RN. A comparison of nasal cytology with sinus $\mathrm{x}$ rays for the diagnosis of sinusitis. American foumal of Rhinolog 1988:2.55-9.

20 Gill FF, Neiburger JB. The role of nasal cytology in the diagnosis of chronic sinusitis. American foumal of Rhinology 1989;3:13-5.

21 Jannert $M$, Andreasson L, Holmer N-G, Lorinc $P$. Ultrasonic examination of the paranasal sinuses. Acta Otolaryngol 1982;389(suppl): 1-51.

22 Pfleiderer AG, Drake-Lee AB, Lowe D. Ultrasound of the sinuses: worthwhile procedure? A comparison of ultrasound and radiography in predicting the findings of proof puncture of the maxillary sinuses. Clin Otolaryngol 1984;9:335-9.

23 Spector SL, Lotan A, English G, Philpot I. Comparison between transillumination and the roentgenogram in diagnosis of paranasal sinus disease. $\exists$ Allergy Clin Immunol 1981;67:22-6.

24 Royal College of Radiologists Working Party. Making the best use of department of clinical radiology: guidelines for doctors. London: RCR, 1993.

25 Zinreich SJ. Paranasal sinus imaging. Otolaryngol Head Neck Surg 1990; 103(suppl):863-9.

26 Druce HM. Emerging techniques in the diagnosis of sinusitis. Ann Allerg 1991;66:132-6.

27 Evans FO Jr, Syndor JB, Moore WE, Moore GR, Manwaring JL, Brill AH et al. Sinusitis of the maxillary antrum. N Engl f Med 1975;293:735-9.

28 Axellson A, Grebelius N, Chidekel N, Jensen C. The correlation between the radiological examination and the irrigation findings in maxillary sinusitis. Acta Otolaryngol 1970;69:302-6.

29 Kovatch AL, Wald E, Ledesma-Medina J, Chiponis DM, Bedingfield B. Maxillary sinus radiographs in children with non-respiratory complaints. Pediatrics 1984;73:306-8.

30 Shankar L, Evans KL, Hawke M, Stammberger H. An atlas of imaging of the paranasal sinuses. London: Martin Dunitz, 1994. ( $£ 75$.)

31 Zinreich J. Imaging of inflammatory sinus disease. Otolaryngol Clin North Am 1993;26:535-47.

32 Kennedy DW, Zinreich SJ, Rosenbaum AE, Johns ME. Functional endoscopic sinus surgery: theory and diagnostic evaluation. Arch Otolaryngol Head Neck Surg 1985;111:576-82.

33 Havas TE, Motbey JA, Gullane PJ. Prevalence of incidental abnormalities on computerised scans of the paranasal sinuses. Arch Otolaryngol Head Nech Surg 1988;114:856-9.

34 Shapiro MD, Som PM, MRI of the paranasal sinuses and nasal cavity. Radio Clin North Am 1989;27:447-75.

35 Zinreich SJ, Kennedy DW, Kumar AJ, Rosenbaum AE, Arrington JA, Johns ME. MR imaging of the normal nasal cycle: comparison with sinus pathology. F Comput Assist Tomogr 1988;12:1014-9.

36 Som PM, Shapiro MD, Biller HF, Sasali C, Lawson W. Sinonasal tumors and inflammatory tissues: differentiation with MR imaging. Radiology 1988; 167:803-8.

37 Zinreich SJ, Kennedy DW, Malat J, Curtin HD, Epstein JI, Huff LC, et al. Fungal sinusitis: diagnosis with CT and MR imaging. Radiology 1988;169: 439-44.

38 Casiano RR. Azithromycin and amoxycillin in the treatment of acute maxillary sinusitis. Am F Med 1991;91(suppl 3A):27-30S.

39 Camacho AE, Cobo R, Otte J, Spector SL, Lemer CJ, Garrison NA, et al. Clinical comparison of cefuroxime axetil and amoxycillin/clavulanate in the treatment of patients with acute bacterial maxillary sinusitis. $\mathrm{Am} \mathcal{f}$ Med 1992;93:271-6.

40 Qvarnberg Y, Kantola O, Salo J, Toivanen M, Valtonen H, Vuori E. Influence of topical steroid treatment on maxillary sinusitis. Rhinology 1992;30: 103-12.

41 Wilson R, Sykes DA, Chan KL, Cole PJ, Mackay IS. Effect of head position on the efficacy of topical treatment of chronic mucopurulent rhinosinusitis. on the efficacy of topica

42 Bergone-Berezin E, Berthelot G, Kafe H, Dournovo. P. Influence of fluidifying agent (bromhexine) on the penetration of antibiotics into respiratory secretions. Int f Clin Pharmacol Res 1985;5:341-4.

43 Mabry RL. Uses and misuses of intranasal corticosteroids and cromolyn. American fournal of Rhinology 1991;5:121-4.

44 Stammberger H. Endoscopic endonasal surgery-concepts in treatment of recurring rhinosinusitis-part 1. Anatomic and pathophysiologic considerations. Otolaryngol Head Neck Surg 1986;94:143-7.

45 Lund VJ. Inferior meatal antrostomy. Fundamental considerations of design and function. $f$ Laryngol Otol Suppl 1988;15:1-18.

46 Messerklinger W. Endoscopy of the nose. Baltimore, MD: Urban and Schwarzenberg, 1978.

47 Stammberger H. Endoscopic endonasal surgery-concepts in treatment of recurring rhinosinusitis-part II. Surgical technique. Otolaryngol Head Neck Surg 1986;94:147-56.

48 Huerter JV Jr. Functional endoscopic sinus surgery and allergy. Otolaryngol Clin North Am 1992;25:2311-8.

49 Kennedy DW. Surgical update. Otolaryngol Head Neck Surg 1990;103(suppl): 884-6.

50 Lanza DC, Kennedy DW. Current concepts in the surgical management of chronic and recurrent acute sinusitis. F Allergy Clin Immunol 1992;90: 505-10

51 Wigand ME. Endoscopic sinus surgery of the paranasal sinuses and anterior skull base. New York: Thieme, 1990:101-12.

52 Stankiewiez JA. Complications of endoscopic intranasal ethmoidectomy. Laryngoscope 1987;97:1270-3.

53 Gamble RC. Acute inflammation of the orbit in children. Arch Ophthalmo 1933;10:483-97.

54 Patt BS, Manning SC. Blindness resulting from orbital complications of sinusitis. Otolaryngol Head Neck Surg 1991;104:789-95.

55 Stammberger H. Functional endoscopic sinus surgery. Philadelphia: Decker, 1991:398-427.

56 Schwietz LA, Gourley DS. Allergic fungal sinusitis. Allergy Proc 1992;13:3-6.

57 Lin PT, Bukachevsky R, Blake M. Management of odontogenic sinusitis with persistent oro-antral fistula. Ear Nose Throat 1991;70:488-90.

58 Muntz HR, Lusk RP. Bacteriology of the ethmoid bullae in children with chronic sinusitis. Arch Otolaryngol Head Neck Surg 1991;117:179-81.

59 Wald ER, Milmoe G, Bowen AD, Ledesma-Medina J, Salamon N, Blueston C. Acute maxillary sinusitis in children. $N$ Engl f Med 1981;304:749-54.

60 McMahon JT. Paranasal sinusitis-geriatric considerations. Otolaryngol Clin North Am 1990;23:1169-77.

61 Kulber DA, Santora TA, Shabot MM, Hiatt JR. Early diagnosis and treatment of sinusitis in the critically ill trauma patient. Am Surg 1991;57:775-9.

62 Cho SY, Choi HY. Opportunistic fungal infection among cancer patients: a ten-year autopsy study. Am F Clin Pathol 1979;72:617-21.

63 Kavanagh K, Hughes WT, Parham DM, Chanin LR. Fungal sinusitis in immunocompromised children with neoplasms. Ann Otol Rhinol Laryngol 1991;100:331-6.

64 Godofsky EW, Zinreich J, Armstrong M, Leslie JM, Weikel CS. Sinusitis in HIV-infected patients: a clinical and radiographic review. $A m \Im$ Med 1992;93:1163-70.

65 Grant A, von Schoenberg M, Grant HR, Miller RF. Paranasal sinus disease in HIV antibody positive patients. Genitourin Med 1993;69:208-12.

\title{
Use of personal medical records for research purposes
}

\author{
Nicholas Wald, Malcolm Law, Tom Meade, George Miller, Eva Alberman, John Dickinson
}

The established practice of doctors using medical records for research purposes is threatened by the recent proposed guidelines from the Department of Health, the BMA, and the European Commission. The European Commission has proposed that explicit consent should be obtained from each patient before his or her medical records can be used; the proposals from the Department of Health and the BMA would require all research that needs access to personal medical records to be submitted to an ethics committee. We believe that these proposals would seriously impair an entire category of research and suggest therefore that another set of guidelines, proposed by a Royal College of Physicians' working group, should be used to modify the proposals. The guidelines of the working group encourage the use of medical records for research and ensure that such use can be made in a confidential manner without causing harm.

The use of medical records for research purposes is a longstanding practice by doctors of virtually all specialties. The value of such a practice has been immense. It is now under threat from proposed guidelines issued by the Department of Health, ${ }^{1}$ from a draft bill governing use and disclosure of personal health information produced by the BMA's Multidisciplinary Professional Working Group, ${ }^{2}$ and a draft data protection directive (SYN 287) from the European Commission. Safeguards to protect the confidentiality of financial and other personal details from unauthorised access by public authorities are being uncritically extended to cover medical records held by doctors. ${ }^{12}$ An entire category of research, which can identify, for example, the side effects of drugs, the effects of toxic substances in the environment, or the safety of medical procedures, would be seriously impaired.

\section{Background}

The use of medical records for research purposes has usually taken two forms: systematic record review and what might be termed "record linkage." Systematic record review is commonplace, carried out, for example, on a consecutive series of patients with 